\title{
Anticoncepção de emergência no Brasil: facilitadores e barreiras
}

\author{
Emergency contraception in Brazil: \\ facilitators and barriers
}

Ellen Hardy 1,2

Graciana Alves Duarte 2

Maria José Duarte Osis 2

Ximena Espejo Arce 1

Magali Possan 2

\footnotetext{
1 Departamento de Tocoginecologia, Faculdade de Ciências Médicas, Universidade Estadual de Campinas. C. P. 6181 , Campinas,SP 13081-970, Brasil. hardy@unicamp.br

2 Departamento de Pesquisas Médico-Sociais, Centro de Pesquisas das Doenças Materno-Infantis de Campinas. C. P. 6181 , Campinas,SP 13081-970, Brasil.
}

Abstract A multi-centered qualitative study was conducted in Brazil, Chile, and Mexico to assess the acceptability of emergency contraception both among potential users and possible providers, authorities, and opinion-makers, and to identify (according to participants' perceptions) factors facilitating or hindering the method's use and the most appropriate strategi es to disseminate information and provide the method. Data were collected through semi-structured interviews,group interviews, and discussion groups, which were tape recorded and transcribed. A thematic analysi s of this material was con ducted. Acceptability of emergency contraception was high among participants, who also felt that there were no barriers towards its acceptance by the population. Partici pants felt that the method's acceptability would be greater if it were included in reproductive health programs, emphasi zing its prescription for emergency situations. Participants highlighted that strategic components in Brazil would betraining of providers and inclusion of the method in family planning services.

Key words Contraception; Abortion; Reproductive Medicine

Resumo Realizou-se um estudo multicêntrico (Brasil, Chile e México), qualitativo, para avaliar a acei tabili dade da anticoncepção de emergência entre potenciais usuárias, possíveis provedores, autoridades e outras pessoas influentes, e identificar, deacordo com a percepção dos participantes,facilitadores e barrei ras para a utilização do método no Brasil. Realizaram-se entrevistas semi-estruturadas, grupais e grupos de discussão, que foram gravados etranscritos para real ização de análi se temática. Os partici pantes manifestaram-se francamente favoráveis à di sseminação da informação, provi são e uso da anti concepção de emergência no Brasil .Consi deraram que não existem barrei ras significativas a sua aceitação pela soci edade brasilei ra em geral, e que se ria mais apropriado adotar-se a estratégi a de inseri-la em programas abrangentes de saúde reprodutiva. O método deveria ser ofereci do como mais uma alternativa contraceptiva, em meio às demais, enfatizando a sua indicação em si tuações de emergência. Além disso, apontou-se como essencial que os profissi onai s de saú de sejam capacitados para proverem a informação eo método.

Palavras-chave Anticoncepção; Aborto; Saúde Reprodutiva 
Introdução

A Anticoncepção de Emergência ( $A E)$ é uma forma de contracepção que pode ser usada pelas mulheres após uma relação sexual desprotegida. Qualquer mulher com risco de gravidez pode precisar deste recurso frente ao uso incorreto ou inconsistente de um método contraceptivo (por exemplo: esquecimento de tomar a pílula, ou de colocar o diafragma), à faIha de métodos (como rompimento do condom ou expulsão do DIU) e/ ou no caso de uma relação sexual imprevista ou violenta (MS, 1996).

No Brasil, o uso de pílulas anticoncepcionais como $A E$ já vem ocorrendo há muitos anos, porém em ambientes restritos, nunca como prática sistematizada. De forma que a sua prescrição pelos profissionais de saúde, no setor público e privado, ainda vem sendo bastante restrita (FEBRASGO, 1996). Em março de 1996, foi realizada em Brasília, uma oficina sobre anticoncepção de emergência, promovida pelo escritório do Population Cound I no Brasil epela Coordenação de Saúde M aterno-Infantil do Ministério da Saúde (MS). Participaram dessa reunião 28 profissionais brasileiros e estrangeiros, com o propósito de definir diretrizes para a introdução da $A E$ na atenção à saúde reprodutiva no Brasil (Population Council, 1996).

Como resultado dessa oficina, o MS incorporou a $A E$ às normas nacionais, incluindo esse método no Manual de Assistência ao Planejamento Familiar, recomendando a utilização do regime Yuzpe (MS, 1996). Em 1998, o Ministério propôs a norma para prevenção e tratamento dos agravos resultantes da violência sexual contra mulheres e adolescentes (MS, 1999), preconizando a oferta de $A E$ às mulheres vítimas de violência sexual. Essa norma entrou em vigor apenas em 1999, depois de bastante polêmica no Congresso Nacional, suscitada por alguns parlamentares que a classificavam como legitimadora do aborto.

Em vista do contexto jurídico e político favorável, já está sendo comercializado nas farmácias um produto específico para AE, dispensando a utilização improvisada dos anticoncepcionais orais que estão no mercado. Porém, ainda não se sabe muito acerca da aceitabilidade desse método na sociedade brasileira em geral e, especificamente, entre potenciais usuárias e provedores. Um estudo sobre conhecimento, atitudes e prática da prescrição da AE pelos ginecologistase obstetras brasileiros apontou que, em geral, eles tinham uma atitude favorável frente à utilização da $A E$, seu conhecimento acerca do método era moderado e poucos deles já haviam prescrito corretamente o regime deYuzpe (Galvão et al., 1999).
Procedimentos metodológicos

Entre 1996 e 1999, o Centro de Pesquisas das Doenças M aterno-Infantis de Campinas (CEMICAMP) participou, juntamente com um centro de pesquisa do Chile e outro do México, do estudo multicêntrico Aceitabilidadeda AE na América Latina. $O$ objetivo desse estudo foi avaliar o conhecimento e a informação sobre $\mathrm{AE}$, bem como a sua aceitabilidade entre potenciais usuárias (mulheres em idade reprodutiva, adolescentes mães, adol escentes sem fiIhos e mães cujos fil lhos estavam na adolescência) dos estratos sócio-econômicos médio e baixo; possíveis provedores da AE (médicos, enfermeiras, professores de adolescentes, ativistas de movimentos de mulheres); formuladores de políticas públicas e autoridades da área da saúde, em nível municipal (Campinas, São Paulo), estadual (Estado de São Paulo) e federal (Ministério da Saúde); e outras pessoas (como padres, jornalistas, farmacêuticos) capazes de influir (influentes) no processo de disseminação da informação e provisão da AE.

Tratou-se de um estudo exploratório, com metodol ogia qual itativa. A informação foi coletada através de grupos de discussão (Krueger, 1994), entrevistas grupais e semi-estruturadas (Fontana \& Frey, 1994). No total houve 38 entrevistas com possíveis provedores da $A E$, formuladores de políticas públicas, autoridades da área da saúde e outras pessoas influentes; oito entrevistas grupais e cinco grupos de discussão com potenciais usuárias. A participação no estudo foi voluntária, mediante assinatura de um Termo de Consentimento Livre e Esclarecido.

As entrevistas e grupos de discussão foram transcritos, procedendo-se à análise temática dos mesmos (Minayo, 1993). Neste trabalho será enfocada a aceitabilidade da AE entre os participantes da pesquisa. O conceito de aceitabilidade adotado pelo estudo foi a aceitação ou não da $A E$ nas representações sociais (percepções e imagens internalizadas) e na ação (decisão e comportamento) dos participantes, entendendo-se que a aceitabilidade resulta da interação de três níveis: macro/ social, institucional e micro/ pessoal.

\section{Resultados}

\section{O pinião geral}

A grande preocupação manifestada em relação à $\mathrm{AE}$, por todas as categorias de participantes, foi a possibilidade de que ela passe a ser usada 
inadequadamente, no lugar de um método anticoncepcional (MAC) de uso cotidiano, regular. Para justificar essa preocupação, os participantes enfatizaram principalmente seu medo de que os adolescentes passem a utilizar a $A E$ rotineiramente, por considerá-la mais fácil, uma vez que eles já apresentam uma certa resistência ao uso consistente de anticoncepção e de prevenção de doenças sexualmente transmissíveis (DST) e Síndrome da Imunodeficiência Adquirida (AIDS). As potenciais usuárias e os provedores foram insistentes em manifestar sua preocupação de que a AE possa ser indevidamente utilizada, provocando prejuízos à saúde das mulheres, como conseqüência do uso repetido de altas doses de hormônios.

De modo geral, os participantes consideraram que todas as mulheres, de qualquer idade, são potenciais usuárias da $A E$, exclusivamente nas situações de emergência, entendidas enquanto possíveis acidentes. No caso de relações sexuais ocasionais, a AE não deveria ser indicada porque as pessoas que já mantêm uma vida sexual deveriam estar sempre preparadas para uma eventualidade. Para essas pessoas, são ideais os métodos de barreira. As potenciais usuárias, porém, ressalvaram que a exceção a essa regra são os adolescentes, dadas as circunstâncias de vida em que se encontram, inclusive de início da vida sexual. Eles teriam justificativa para usar a AE frente a uma relação ocasional, inesperada. Outro ponto enfatizado apenas pelas potenciais usuárias foi que as pessoas casadas não deveriam usar a $A E$, uma vez que essa condição pressupõe que elas estão sujeitas a procriar.

\section{Anticoncepção de emergência e aborto}

De modo geral, os entrevistados em todas as categorias concordaram que a $\mathrm{AE}$ age evitando uma gravidez, por impedir a ovulação, a fecundação ou a nidação. Apenas um professor de instituição católica de ensino afirmou que a AE induz o aborto, e outro, de instituição evangélica, manifestou dúvidas com respeito ao método ser abortivo ou não, por não estar convicto sobre o mecanismo de ação. Para ele, se a AE agir sobre a fecundação estará impedindo a gravidez, caso contrário, agindo apenas sobrea nidação, induziria o aborto.

Embora a tendência dos entrevistados fosse considerar que a AE não induz o aborto, mas impede a gravidez, eles enfatizaram que o conceito de gravidez varia de indivíduo para indivíduo e, em função disso, das perspectivas individuais e/ ou grupais, algumas pessoas e/ou grupos poderão consi derar que a AE é abortiva.
As potenciais usuárias frisaram que a associação entre AE e aborto vai depender dos vaIores morais e religiosos de cada pessoa e da importância dos mesmos na vida delas. Entre os médicos não ginecologistas, mas possíveis provedores da AE por atuarem em serviços de atenção primária e/ ou de urgência, houve quem manifestasse a opinião de que o uso desse método poderia significar a indução de um aborto. Mesmo entre ginecologistas foi utilizado o qualificativo "microabortivo", argumentandose que o método pode eliminar o óvulo fecundado. Apenas um profissional disse que não Ihe era clara a diferença entre o uso da AE e a indução do aborto, apesar de ter lido a norma técnica do MS sobre a anticoncepção de emergência.

\section{Aceitação}

Houve consenso de que toda a população tem direito a saber que existe o recurso da AE. As pessoas influentes ressaltaram que isto é uma questão de cidadania, de se respeitarem os direitos dos cidadãos. Todos os participantes consideraram que no Brasil as barreiras para a introdução e uso da AE são relativas, estando associadas muito mais a perspectivas individuais do que a resistências estruturais da sociedade como um todo. Por exemplo, o medo de que ela seja utilizada de forma inadequada, sendo entendida como método de rotina, poderia fazer com que não se divulgasse o método. Isto foi enfatizado especial mente em relação aos adolescentes, para os quais se acrescentou como possível barreira a oposição dos pais a que eles recebam informação e tenham acesso à AE.

Os participantes enfatizaram que, do ponto de vista legal e dos programas de saúde, não há barreiras porque a AE está legitimada pela Lei de Planejamento Familiar e pela norma específica do MS. Isto dá amparo legal e técnico aos profissionais de saúde para oferecerem o método, tornando-o mais aceitável. As pessoas influentes salientaram que o contexto social no Brasil é favorável a que os serviços públicos de saúde disseminem informação e provejam a AE. Entretanto, os participantes ressaltaram que, apenas a existência da norma, não garante que as mulheres terão acesso à $A E$. É necessário criar mecanismos que acompanhem a aplicação da norma, de fato. Nesse sentido, as pessoas influentes e os provedores, de modo geral, apontaram como barreira em potencial as dificuldades de infra-estrutura dos serviços públicos de saúde do país, especialmente a falta de insumos contraceptivos e de pessoal 
devidamente capacitado para informar e prover a AE. Na perspectiva desses entrevistados, a capacitação do pessoal da área de saúde para prover o método deve enfocar a parte técnica mas também outros aspectos inerentes à prestação de serviços com boa qualidade de atenção.

Finalmente, foi dito que o processo de disseminação de informação e provisão da AE será facilitado se os meios de comunicação de massa forem utilizados cuidadosamente, para não suscitar reações de possí veis opositores. Para os provedores de serviços foi considerado essencial que a distinção entre AE e RU 486 fique bem clara, e que a AE seja apresentada como mais uma opção contraceptiva, incluída em um amplo programa, que abranja tanto a educação sexual quanto a informação e provisão de contracepção.

\section{Comentários}

Os resultados apresentados permitiram verificar que os participantes do estudo percebiam a $A E$ como um recurso desejável, de modo geral aceitável por toda a população, que deve estar acessível através de programas amplos de saúde sexual e reprodutiva. Um elemento fundamental para essa perspectiva é a legitimidade social atribuída à AE com base em seu res- paldo na Lei sobre Planejamento Familiar (Brasil, 1997) e em sua normatização pelo Ministério da Saúde para aplicação nos serviços públicos em todo o país (MS, 1996). Enfatizou-se, porém, a necessidade de que a provisão desse método seja controlada pel os serviços de saúde. Todas as pessoas deveriam saber da existência da $A E$, mas não deveria ser possível usála sem uma avaliação profissional, preferencialmente realizada por médico. A mesma perspectiva foi observada em uma sociedade distinta, entre médicos ingleses (Ziebland, 1999).

É possível perceber portanto, a partir das observações dos participantes deste estudo, que a disseminação de informações sobre a $A E$ deve ser bastante cuidadosa para não comprometer a sua aceitação. Deve-se dissociá-la o máximo possível da polêmica sobre o aborto, ressaltando, ao mesmo tempo, seu caráter contraceptivo e emergencial. Não se deveria permitir que ela seja apresentada como uma solução mágica para evitar o problema da gravidez indesejada, como se pudesse substituir o uso regular de métodos anticoncepcionais. Em vista disso, é fácil perceber porque os participantes recomendaram que os meios de comunicação sejam usados com cautela para divulgar a $A E$, e que, ao contrário, os serviços de saúde é que devem ser as principais fontes de informação, através de programas voltados a ações educativas.

\section{Agradecimentos}

Trabalho realizado no Departamento de Pesquisas Médico-Sociais do Centro de Pesquisas das Doenças Materno-Infantis de Campinas (CEMICAMP) da Universidade Estadual de Campinas. Projeto parcialmente financiado por Mellon Foundation, através da Organização Mundial da Saúde. 


\section{Referências}

BRASIL, 1997. Lei ordinária número 9263 de 12 de janeiro de 1996. Regula o parágrafo 7 do artigo 226 da Constituição Federal, que trata do planejamento familiar, estabelece penalidade e dá outras providências. Brasília: Diário Oficial da União, p. 17989, col. 1, 20 ago.

FEBRASGO (Federação Brasileira das Sociedades de Ginecologia e Obstetrícia), 1996. Pílula pós-coital: Fator decisivo na prevenção à gravidez indesejada. Jornal da Febrasgo, 25:6-7.

FONTANA, A. \& FREY, J. H., 1994. Interviewing: The art of science. In: Handbook of Qualitative Research (N. K. Denzin \&Y. S. Lincoln, ed.), pp. 361376, Thousand Oaks: Sage Publications.

GALVÃO, L.; DIAZ, J.; DIAZ, M.; OSIS, M. J. D.; ELLERTSON, C. \& CLARK, S., 1999. Emergence contraception: Knowledge, attitudes, and practices among Brazilian obstetrician-gynecologists. International Family Planning Perspectives, 25: 168-171.

KRUEGER, R. A., 1994. Focus Groups - A Practical Guide for Applied Research. 2nd Ed. Thousand Oaks: Sage Publications.
MINAYO, M. C. S., 1993. O Desafio do Conhecimento: Pesquisa Qualitativa em Saúde. 2a Ed. Rio de Janeiro: Editora Hucitec/ ABRASCO.

MS (Ministério da Saúde), 1996. Anticoncepção de emergência. In: Assistência ao Planejamento Familiar (Serviço de Assistência à Saúde da Mulher, org.), pp. 121-125, Brasília: Secretaria Executiva, Coordenação de Saúde da Mulher, Ministério da Saúde.

MS (Ministério da Saúde), 1999. Prevenção e Tratamento dos Agravos Resultantes da Vi olência Sexual Contra Mulheres e Adolescentes - Norma Técnica. Brasília: MS

POPULATION COU NCIL, 1996. Relatório Final da I Oficina Brasileira sobre a Anti concepção de Emergência: Um Grupo deConsulta para o seu Uso no Brasil. Brasília: Population Council/Coordenação Materno-Infantil, Ministério da Saúde do Brasil. (mimeo.)

ZIEBLAND, S., 1999. Emergency contraception: An anomalous position in the family planning repertoire? Social Science and Medicine, 49:1409-1417. 